\title{
Blocked Autophagy Sensitizes Resistant Carcinoma Cells to Radiation Therapy
}

\author{
Anja Apel, ${ }^{1,3}$ Ingrid Herr, ${ }^{3}$ Heinz Schwarz, ${ }^{2}$ H. Peter Rodemann, ${ }^{1}$ and Andreas Mayer ${ }^{4}$ \\ ${ }^{1}$ Division of Radiobiology and Molecular Environmental Research, University of Tuebingen; ${ }^{2}$ Max-Planck Institute for Developmental \\ Biology, Tuebingen, Germany; ${ }^{3}$ Molecular OncoSurgery, Department of General Surgery, University of Heidelberg and German Cancer \\ Research Center, Heidelberg, Germany; and ${ }^{4}$ Department of Biochemistry, University of Lausanne, Epalinges, Switzerland
}

\begin{abstract}
Autophagy or "self eating" is frequently activated in tumor cells treated with chemotherapy or irradiation. Whether autophagy represents a survival mechanism or rather contributes to cell death remains controversial. To address this issue, the role of autophagy in radiosensitive and radioresistant human cancer cell lines in response to $\boldsymbol{\gamma}$-irradiation was examined. We found irradiation-induced accumulation of autophagosomes accompanied by strong mRNA induction of the autophagy-related genes beclin 1, atg3, atg4b, atg4c, atg5, and atg12 in each cell line. Transduction of specific targetsiRNAs led to down-regulation of these genes for up to 8 days as shown by reverse transcription-PCR and Western blot analysis. Blockade of each autophagy-related gene was associated with strongly diminished accumulation of autophagosomes after irradiation. As shown by clonogenic survival, the majority of inhibited autophagy-related genes, each alone or combined, resulted in sensitization of resistant carcinoma cells to radiation, whereas untreated resistant cells but not sensitive cells survived better when autophagy was inhibited. Similarly, radiosensitization or the opposite was observed in different sensitive carcinoma cells and upon inhibition of different autophagy genes. Mutant p53 had no effect on accumulation of autophagosomes but slightly increased clonogenic survival, as expected, because mutated p53 protects cells by conferring resistance to apoptosis. In our system, short-time inhibition of autophagy along with radiotherapy lead to enhanced cytotoxicity of radiotherapy in resistant cancer cells. [Cancer Res 2008;68(5):1485-94]
\end{abstract}

\section{Introduction}

Macroautophagy, usually referred as autophagy, is becoming an important area in cancer research. There are several recent reviews describing the relationship of autophagy and apoptosis signaling during cancer therapy (1-4), and a number of antineoplastic therapies have been observed to induce autophagy in human cancer cell lines (5-8). Autophagy is a genetically programmed, evolutionarily conserved process that is involved in degradation of long-lived cellular proteins and organelles and in the turnover of cytoplasmic organelles such as mitochondria, peroxisomes, and

Note: Supplementary data for this article are available at Cancer Research Online (http://cancerres.aacrjournals.org/).

H.P. Rodemann and A. Mayer contributed equally to this work.

Requests for reprints: Ingrid Herr, University of Heidelberg and German Cancer Research Center-G403, Molecular OncoSurgery, Im Neuenheimer Feld 365, 69120 Heidelberg, Germany. Phone: 49-6221-56-38354; Fax: 49-6221-56-6119; E-mail: i.herr@dkfz.de.

(C)2008 American Association for Cancer Research.

doi:10.1158/0008-5472.CAN-07-0562 endoplasmic reticulum (9). In contrast, the proteasome is involved in the degradation of short-lived proteins including stress response and DNA damage repair. During autophagy, cytoplasmic constituents are enclosed by an isolation membrane resulting in the formation of an autophagosome. This is a double-membraned vesicle, which encapsulates cytoplasm and organelles. The outer membrane of the autophagosome fuses with a lysosome. The inner membrane, called autophagic body, and its protein and organelle contents are then degraded by lysosomal enzymes and recycled (9-12). The formation of the double-membraned vesicle is a complex process involving 16 autophagy-related proteins (13) Beclin 1 is such an autophagy-related protein and part of the class III PI3 kinase complex playing an important role in production of PI-3-phosphate. This may be essential for sorting of autophagosomal components to preautophagosomal structures as well as for lysosomal enzyme transport. The Beclin-PI3-kinase complex localizes to the cytosol and the trans-Golgi network (14-17). Other proteins such as Atg5 and Atg12 are part of an ubiquitin-like conjugation system mediating large membrane-associated protein complexes that are required for autophagy $(9-11,18,19)$. Atg3 and Atg4, together with phosphatidylethanolamine, are important for the function of a different ubiquitin-like conjugation system mediating a protein/phospholipid complex that localizes to autophagosomal membranes (9-11).

Whether autophagy induced by cancer therapy contributes to tumor cell death or represents a mechanism of resistance to therapy-mediated cell death remains uncertain. On nutrient deprivation, autophagy is increased, allowing the cell to degrade proteins and organelles and, thus, obtain a source of macromolecular precursors, such as amino acids, fatty acids, and nucleotides, which would not be available otherwise (4). Programmed cell death can also be triggered by nutrient deprivation, and autophagy induced in this context can serve to inhibit apoptosis. Thus, when autophagy is prevented under these conditions, the cells undergo apoptosis (20-22). Consequently, when tumor cells are starved, autophagy may stop them from dying by inhibiting apoptosis. In a tumor, this may mean that autophagy keeps tumor cells alive when limited angiogenesis leads to nutrient deprivation and hypoxia; therefore, increased autophagy might promote the growth of solid tumors, whereas reduced autophagy might provide a useful way to limit tumor growth (4).

Conversely, persistent activation of autophagy can also lead to programmed cell death (23), and autophagy can stimulate cancer, depending on the context. For example, Beclin 1 induces autophagy when overexpressed and inhibits tumor growth, whereas heterozygous disruption of beclin 1 promotes tumorigenesis in mice $(24,25)$. These animal studies are confirmed by observations in patients because beclin 1 is frequently monoallelically lost in human breast, ovarian, and other tumors (7). Correspondingly, antiapoptotic Bcl-2 proteins, involved in resistance of tumors, have 
been shown to directly interact with Beclin 1 to inhibit autophagy in yeast and mammalian cells. This antiautophagy function of Bcl-2 may help maintain autophagy at low levels that are compatible with cell survival, rather than cell death (26).

Inactivation of cell-death pathways is a central component of cancer progression, (27) and $p 53$, a tumor suppressor mutated in $\sim 50 \%$ of all tumors (28), is a critical mediator of cell death. Although a role for $p 53$ in apoptosis is well-established, a direct link to autophagy has been suggested (29) but is not examined in detail.

Although many anticancer agents have been reported to induce autophagy, including tamoxifen, rapamycin, arsenic trioxide, temozolomide, histone deacetylase inhibitors, ionizing radiation (7), vitamin D analogues (30), and etoposide (31), it is highly controversial whether autophagy really contributes to the effectiveness of cancer therapy. Because autophagy occurs in tumor cells before their demise, it does not necessarily follow that autophagy killed the cells; instead, autophagy may be a mechanism by which the cell is trying to survive. To address this issue, one must show that inhibition of autophagy causes increased tumor cell clonogenic growth after anticancer treatment. In most of the examples cited above, this has not been shown; we only know that the drug induced autophagy and then the cells died. Therefore, in this study, the six major autophagy-related genes beclin 1, atg3, atg $4 b$, atg $4 c$, atg 5 , and atg12 were inhibited by specific target-siRNA oligonucleotides in human-resistant and human-sensitive carcinoma cells; and the effect on clonogenic survival of irradiated cancer cells was evaluated.

\section{Materials and Methods}

Cell lines and treatment. MDA-MB-231 breast cancer cells with mutant p53 proteins (32), HTB43 pharyngeal cancer cells with mutant p53 (33), HTB35 cervical squamous cell carcinoma cells with mutant p53 (33), A549 lung cancer cells with wild-type p53, A549 cells transfected with an Ectysone-inducible mutant $p 53$ construct (34), and SW707 rectum carcinoma cells (35) were used. For development of therapy resistance in vitro, parental SW707 cells were continuously cultured with increasing concentrations of cisplatin starting from $0.5 \mathrm{up}$ to $3.5 \mu \mathrm{g} / \mathrm{mL}$ for 6 months. Tumor cells were cultured in DMEM (Life Technologies) supplemented with $10 \%$ fetal bovine serum (HyClone, Perbio Science). Cells were irradiated using a Gulmay Medical RS225 Research System (200 kV; 15 mA; dose rate, $3 \mathrm{~Gy} / \mathrm{min})$. Rapamycin was from Axxora.

Transfection of siRNA. siRNA oligonucleotides were from Dharmacon. Transfection of siRNA was performed with Lipofectamine 2000 according to the protocol provided by the manufacturer (Invitrogen). siGENOME SMARTpool reagents (Dharmacon) were diluted with Opti-MEM I reduced serum (Invitrogen). Antibiotic-free medium was used for experiments with transfected cells.

RNA isolation and conventional reverse transcription-PCR. Total cellular RNA from 1 to $5 \times 10^{5}$ cells was prepared with the Classic Line E.Z.N.A. total RNA kit (PeqLab), and reverse transcription-PCR (RT-PCR) was performed with QIAGEN OneStep RT-PCT kit (QIAGEN) using GeneAmp PCR System 9700 (Applied Biosystems). Primer sequences and conditions are available upon request.

LightCycler RT-PCR. For quantitative LightCycler-PCR, $1 \mu \mathrm{g}$ RNA per sample was converted to cDNA using the First Strand cDNA Synthesis kit (Roche Diagnostics $\mathrm{GmbH}$ ). cDNA synthesis was performed in a GeneAmp PCR System $2400\left(25^{\circ} \mathrm{C}, 10 \mathrm{~min} ; 42^{\circ} \mathrm{C}, 60 \mathrm{~min}\right.$; and $\left.99^{\circ} \mathrm{C}, 5 \mathrm{~min}\right)$. cDNA was diluted 1:5 and used for the LightCycler-PCR. Primer sequences and conditions are available upon request. Data were normalized based on gapdh, and the relative gene expression was calculated by RNA amounts.

Protein isolation and Western blot analysis. Whole cell extracts were prepared by a standard protocol, and proteins were detected by Western blot analysis using polyclonal (mouse) anti-Beclin 1 antibody or polyclonal (rabbit) anti- $\beta$-actin antibody (Sigma-Aldrich). Goat anti-mouse IgG or goat anti-rabbit IgG (Pierce) secondary antibody, conjugated to horseradish peroxidase and enhanced chemiluminescence detection systems (Super Signal West Femto; Pierce) were used for detection.

Electron microscopy. Cells were fixed with $2.5 \%$ glutardialdehyde, postfixed in $1 \%$ osmium tetroxide in PBS for $1 \mathrm{~h}$ on ice, rinsed with doubledistilled water, and then treated with $1 \%$ aqueous uranyl acetate for $1 \mathrm{~h}$ at $4^{\circ} \mathrm{C}$. Samples were dehydrated by graded series of ethanol and embedded in Epon. The bottom of the 24-well tissue plates was sawed, and the plastic was blast from the Epon by temperature shifts between liquid nitrogen and hot water. Ultrathin sections were stained with uranyl acetate and lead citrate, and viewed in a Philips CM10 electron microscope at $60 \mathrm{kV}$.

Immunofluorescence. Cells were fixed with $4 \%$ paraformaldehyde for $10 \mathrm{~min}$ at $37^{\circ} \mathrm{C}$ followed by washing in PBS. Cover slips were embedded with MoWiol. Cells were viewed with a Zeiss fluorescence microscope on 63 objective (excitation, $488 \mathrm{~nm}$; emission, $512 \mathrm{~nm}$ ). For vesicle quantification, 50 cells per treatment were examined, and the vesicles/cell were counted. Three independent experiments were performed.

Colony forming assays. Cells were seeded at a density of $1 \times 10^{5}$ in 24-well tissue culture plates $\left(2.0 \mathrm{~cm}^{2}\right.$; BD Falcon $)$ and were transfected $24 \mathrm{~h}$ later. Forty-eight hours posttransfection, the cultures were treated with single-dose irradiation $(0,1,2,3$, or $4 \mathrm{~Gy})$; and $200,500,600,700$, or 800 cells per well were seeded in 6-well tissue culture plates $\left(9.6 \mathrm{~cm}^{2}\right.$; BD Falcon) and incubated for 10 days without changing medium. For colony formation assays, after fractionated irradiation, cells were seeded in 12-well tissue culture plates $\left(3.8 \mathrm{~cm}^{2}\right.$; BD Falcon) and transfected $24 \mathrm{~h}$ later. Forty-eight hours posttransfection, the first dose of $1 \mathrm{~Gy}$ was applicated, followed by additional daily doses of $1 \mathrm{~Gy}$, for a total of $4 \mathrm{~Gy}$. Immediately after irradiation, cells were seeded in 6-well tissue culture plates and incubated for 10 days. For determination of colony formation from single cells, cultures were fixed (3.7\% paraformaldehyde and 70\% ethanol) and stained with $0.05 \%$ Coomassie Blue. The number of colonies with $>50$ cells was counted under a dissecting microscope. The percentage of cell survival was calculated (plating efficiency of control nonsense-siRNA-transfected cultures, $100 \%$ or relative survival rate).

Viability assay. Cells were seeded at a density of $5 \times 10^{4} / \mathrm{mL}$ in 96 -well tissue culture plates (BD Falcon). Twenty-four hours later, cells were treated with cisplatin or were $\gamma$-irradiated. Seventy-two hours after treatment, $10 \mu \mathrm{L}$ of $12 \mathrm{mmol} / \mathrm{L}$ 3-(4,5-dimethylthiazol-2-yl)-2,5-diphenyltetrazolium bromide (MTT) solution was added to each well, and the plates were incubated at $37{ }^{\circ} \mathrm{C}$ for $4 \mathrm{~h}$. Medium was removed and $200 \mu \mathrm{L}$ DMSO per well was added. Absorbance was measured at $560 \mathrm{~nm}$ with a Victor plate reader. The percentage of cell survival was calculated relative to control cells, which were set to $100 \%$.

Statistical evaluations. For Colony forming assays and MTT measurements, statistical evaluations are presented as mean \pm SE. Data were analyzed using the Student's $t$ test for statistical significance. $P$ values were considered significant if $<0.05$.

\section{Results}

Irradiation induces autophagy. Accumulation of autophagosomes was examined in MDA-MB-231 breast, HTB43 pharyngeal, A549 lung, and HTB35 cervical cancer cell lines before and 1, 2, 4, 8, 12 , and $24 \mathrm{~h}$ after $\gamma$-irradiation. Transmission electron microscopy revealed formation of autophagic vesicles between 1 and $12 \mathrm{~h}$ after irradiation as exemplified for MDA-MB-231 and HTB43 cells (Fig. 1A). Degradation of the vesicles is obvious from the darker and condensed form, leading to clearance at $24 \mathrm{~h}$ after irradiation. To further highlight formation of autophagosomes, we examined the cells by microtubule-associated protein 1 light chain 3 (short LC3p), which is a general marker for autophagic membranes. During autophagosome formation, this protein is conjugated to phosphatidylethanolamine, associates to the vesicle membrane, and remains on the membrane, even after autophagosomes are 
Figure 1. Induction of autophagy by irradiation. A, MDA-MB-231 and HTB43 cells were irradiated (2 Gy) and 1, 2, 4, 8,12 , and $24 \mathrm{~h}$ later, cells were fixed, treated as described in the Materials and Methods section, and viewed in a Philips CM10 electron microscope at $60 \mathrm{kV}$. The images were taken with a $\times 5,000$ objective (scale bars, $1 \mu \mathrm{mol} / \mathrm{L}$ ). Arrows, double-membrane vesicles. $B$, MDA-MB-231 were transfected with GFP-LC3 plasmid together with a nonsense control-siRNA. Two days after transfection, cells were left untreated $(C O)$, were incubated with rapamycin (22 $\mu \mathrm{mol} / \mathrm{L})$, or were $\gamma$-irradiated (10 Gy). Seventy-two hours after rapamycin and $4 \mathrm{~h}$ after $\gamma$-irradiation, control and treated cells were fixed, and fluorescence was viewed with a Zeiss fluorescence microscope using a $\times 63$ objective (excitation, $488 \mathrm{~nm}$; emission, $512 \mathrm{~nm}$ ) $C$, MDA-MB-231 and HTB43 cells were irradiated with different doses $(0,2,4$, and $10 \mathrm{~Gy}$ ). Four hours after irradiation, cellular RNA was isolated and converted into cDNA. Expression of beclin 1, atg3, atg $4 b$, atg $4 c$, atg5, and atg 12 was quantified by LightCycler-PCR using specific primer oligonucleotides available upon request. RNA expression was normalized against gapdh. D, MDA-MB-231 and HTB43 cells were irradiated with 2 Gy. $0.5,1,2,4,8$, and $24 \mathrm{~h}$ after irradiation, RNA expression was examined as described above. Points, mean of two independent experiments.

\section{A Irradiation}

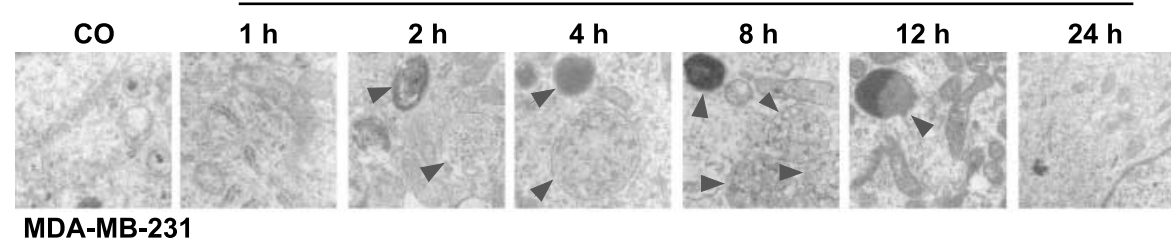

MDA-MB-231

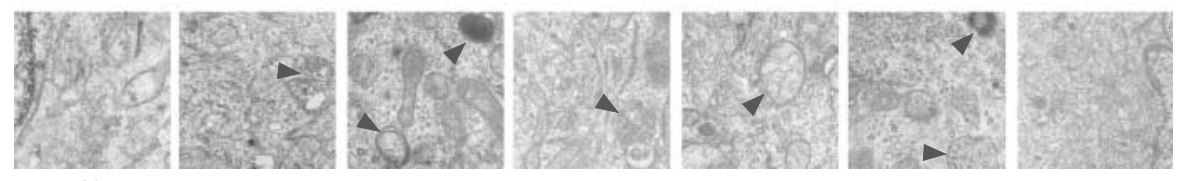

HTB43

B
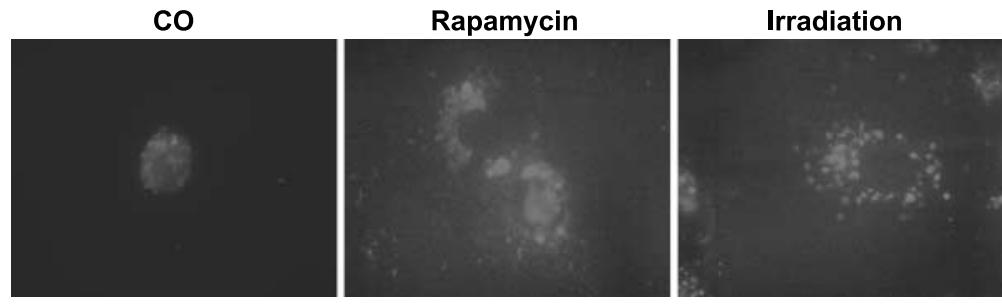

MDA-MB-231

C

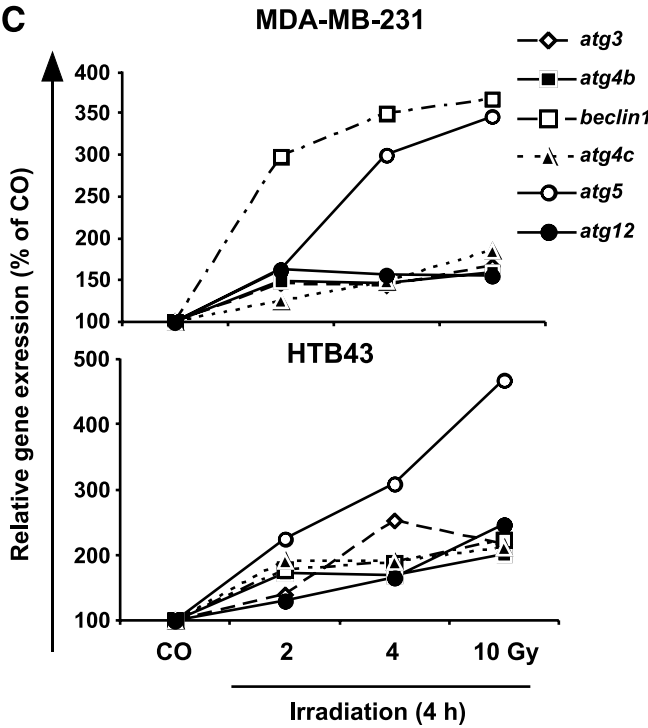

D MDA-MB-231
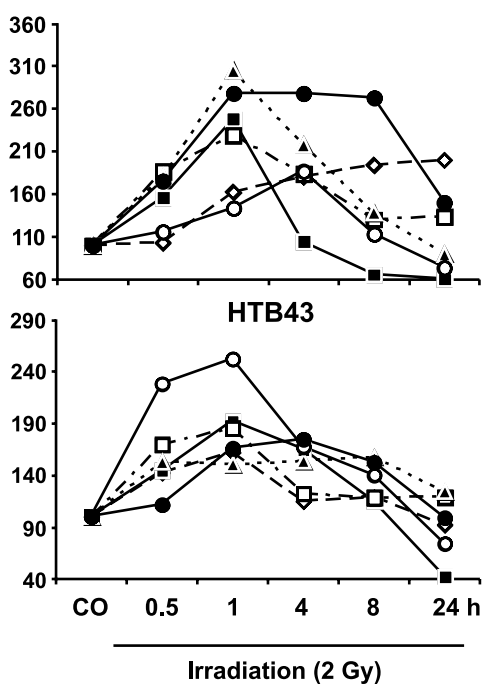

completely formed $(36,37)$. Fusion of GFP with $L C 3$ provides a fluorescent marker for autophagy, which was transfected in the cells. Two days later, cells were irradiated and autophagosome formation was visualized after incubation for additional $4 \mathrm{~h}$ by fluorescence microscopy. Although a basal green fluorescent protein (GFP) fluorescence could be detected in control cells (Fig. $1 B$ ), the intensity was strongly increased by rapamycin, which serves as a positive control for autophagy. Likewise, $\gamma$-irradiation generated vesicles with enhanced size and fluorescence intensity.

To examine whether irradiation induces expression of autophagy-related genes, dose-response experiments were performed. HTB43, MDA-MB-231, A549, and HTB35 cells were left untreated or were $\gamma$-irradiated with 2 , 4, or $10 \mathrm{~Gy}$. RNA was isolated $2 \mathrm{~h}$ later, and gene expression was quantified by LightCycler-PCR and normalized against gapdh. In all cell lines, a dose-dependent increase in expression of autophagy-related genes could be observed (Fig. $1 C$; Supplementary Fig. S1 $A$ ). The steady-state basal expression levels of the autophagic genes were comparable among the four different cell lines (data not shown). As the therapeutic dose of 2 Gy provoked a clear induction of autophagy, this dose was used for most of the following experiments. In a time-response experiment, cells were $\gamma$-irradiated with $2 \mathrm{~Gy}$, indicating that RNA expression of autophagy genes was induced as soon as $30 \mathrm{~min}$ after irradiation with partially still enhanced levels at $24 \mathrm{~h}$ (Fig. $1 D$; Supplementary Fig. S1B)

Inhibition of autophagy-related genes prevents irradiationinduced accumulation of autophagosomes. For inhibition of autophagy, specific target-siRNA oligonucleotides toward beclin 1, 
atg3, atg 4b, atg4c, atg5, and atg12 were transfected in MDA-MB231, A549, HTB43, and HTB35 cancer cell lines. Down-regulation and duration of inhibition were analyzed by OneStep-RT-PCR. Significant inhibition of RNA expression of all genes was found $24 \mathrm{~h}$ after transfection, with an average duration of strong inhibition between 5 and 8 days after transfection. At later time points, the expression started to increase and dropped back to basal expression at day 14. Representative data are shown (Fig. 2A). Similarly, protein expression was inhibited, as analyzed by Western blot (Fig. $2 B$ ). The specific beclin 1 siRNA oligonucleotides totally abolished protein expression of Beclin 1 at day 2, 4, and 6 after transfection, which started to increase at day 9 and reached basal levels at day 11 after transfection. In contrast, the control nonsensesiRNA oligonucleotide had no effect on Beclin 1 protein expression at any time point.

Next, involvement of expression of autophagy-related genes in $\gamma$-irradiation-induced autophagosome accumulation was examined.

A

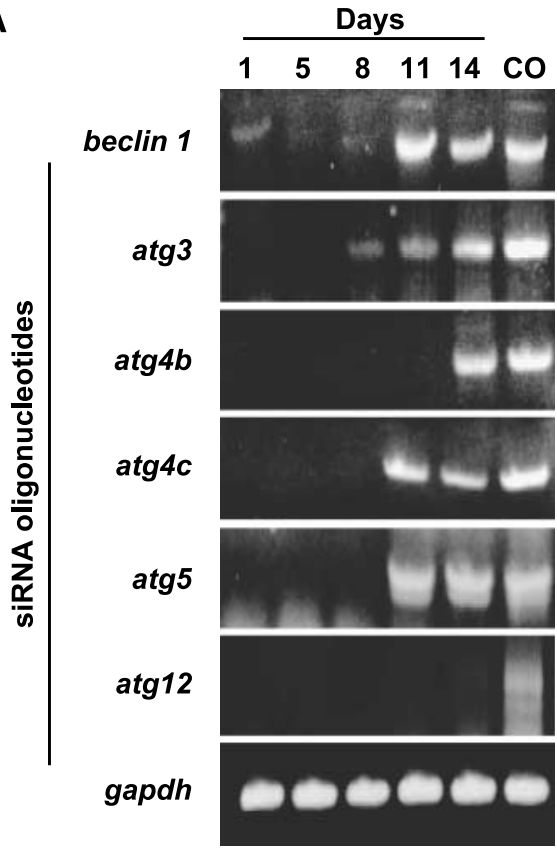

B ns-siRNA $\begin{array}{llllllllllll}2 & 4 & 6 & 9 & 11 & 14 & 2 & 4 & 6 & 9 & 11 & 14\end{array}$

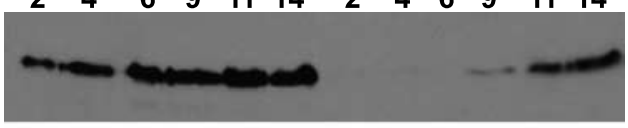

BECLIN 1

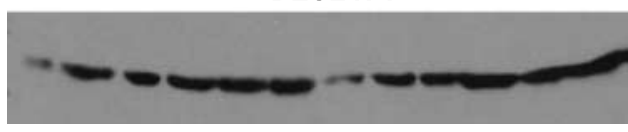

$\beta$-ACTIN
MDA-MB-231 and HTB43 cell lines were treated with Lipofectamine transfection reagent alone or together with control nonsense-siRNA or with specific target-siRNA. Two days after transfection, cells were $\gamma$-irradiated or were left untreated in the controls. Two hours after $\gamma$-irradiation, cells were fixed and examined by transmission electron microscopy. Formation of autophagosomes was observed in irradiated control cells, whereas nonirradiated cells or irradiated cells transfected with the specific siRNA oligonucleotides contained a strongly diminished amount of autophagosomes, as shown for MDA-MB-231 cells (Fig. 3A). Similar results were obtained with HTB43 cells (data not shown). For quantification of the inhibitory effect of specific siRNA oligonucleotides, we cotransfected GFP-LC3 plasmid and counted the formation of autophagic GFP-LC3 fluorescent vesicles per cell according to a recent work (38). Irradiation significantly increased the number of autophagic vesicles in control cells of MDA-MB-231 cells with mutant $p 53$, A549 cells with wild-type, and A549 cells with mutant p53 (Fig. $3 B$ ). No difference in induction was seen between cells with functional and nonfunctional $p 53$. In contrast, upon inhibition of autophagy-related genes by specific targetsiRNA oligonucleotides, only a limited number of $\gamma$-irradiated cells was able to form autophagosomes irrespective of the $p 53$ status.

Clonogenic survival of tumor cells after single dose irradiation. To examine whether inhibition of autophagosome accumulation may sensitize cancer cells for $\gamma$-irradiation or not, we performed colony forming assays. First, the radiosensitivity of HTB43, MDA-MB-231, A549, and HTB35 cells was analyzed using cells transfected with control nonsense-siRNAi oligonucleotides and after $\gamma$-irradiation with single doses of $1,2,3$, or $4 \mathrm{~Gy}$. By this way, we found that HTB43 and MDA cells are relatively resistant to irradiation, whereas A549 and HTB35 cells reacted more sensitively (Fig. 4A). Cells were transfected with specific target-siRNAi oligonucleotides to inhibit autophagy-related genes, followed by $\gamma$-irradiation with single doses of 1 to 4 Gy 2 days later. Ten days after irradiation, colony formation was evaluated (Fig. 4B; Supplementary Fig. S2; Table 1). The most pronounced effect of blocked autophagy-related genes was seen in highly resistant HTB43 cells. Inhibition of the atg3, atg $4 b$, atg $4 c$, and atg12 genes resulted in a lower survival after irradiation. Similar results were obtained after inhibition of atg12 in MDA and HTB35 cells, after inhibition of atg5 in MDA-MB-231 cells, and after inhibition of atg3 in HTB35 cells; whereas inhibition of atg4c in MDA-MB-231 and HTB35 led to a faster growth. In radioresistant cell lines, inhibition of beclin 1 resulted in inhibition of clonogenicity at 1 Gy but in an increase at 2 and $3 \mathrm{~Gy}$. In contrast, inhibition of beclin 1 enhanced clonogenicity in sensitive cancer cell lines. These results suggest sensitization of radioresistant cancer cells by inhibition of atg3, atg $4 b$, atg5, and atg12; whereas inhibition of beclin 1 and $\operatorname{atg} 4 c$ may also result in radioprotection, dependent on the radiation dose used.

Clonogenic survival of tumor cells after fractionated irradiation. The effect of fractionated irradiation (exposure to one daily fraction of 1 Gy at 4 consecutive days) was analyzed because cells can retire from sublethal damage in the times between the fractions. During fractionated radiation, the dose is not applicated once but in diverse portions, leading to a dose saturation during the course of the experiment. This experimental setting resembles more the clinical situation. However, the different setting also changed the outcome on clonogenic survival (Fig. $5 A$; Supplementary Fig. S3; Table 1). Although inhibition of atg3 and $a t g 4 b$ had the same effect as observed for single-dose irradiation oligonucleotides. $A$, cells were transfected with specific siRNA oligonucleotides directed toward beclin 1, atg3, atg $4 b$, atg $4 c$, atg5, and atg12. Cellular mRNA was isolated $1,5,8,11$, and $14 \mathrm{~h}$ after transfection. RNA expression was examined by RT-PCR and gapdh served as internal standard. $B$, protein was isolated 2, 4, 6, 9, and $11 \mathrm{~h}$ after transfection, and Beclin 1 protein expression was analyzed by Western blot. $\beta$-Actin served as loading control. 
Figure 3. Autophagosome accumulation is inhibited in siRNA-transfected cells. A, MDA-MB-231 cells were transfected with nonspecific $(n s)$ or specific RNAi oligonucleotides as indicated. Forty-eight hours later, cells were left untreated or were $\gamma$-irradiated (2 Gy). Two hours after irradiation, cells were fixed and examined as described in Fig. 1A. B, A549 cells with wild-type p53 and A549 cells transfected with an Ectysone-inducible mtp53 were treated with Ponasteron to induce expression of mutated $p 53$. MDA-MB-231, A549, and A549 mtp53 cells were transfected with nonsense or specific targeting siRNA oligonucleotides as described above. Forty-eight hours after transfection, cells were $\gamma$-irradiated (10 Gy) and fixed $6 \mathrm{~h}$ later. Autophagic vesicles were counted in 50 cells per treatment, and the median numbers of autophagosomes per viable cell are shown. Columns, mean of three independent experiments.

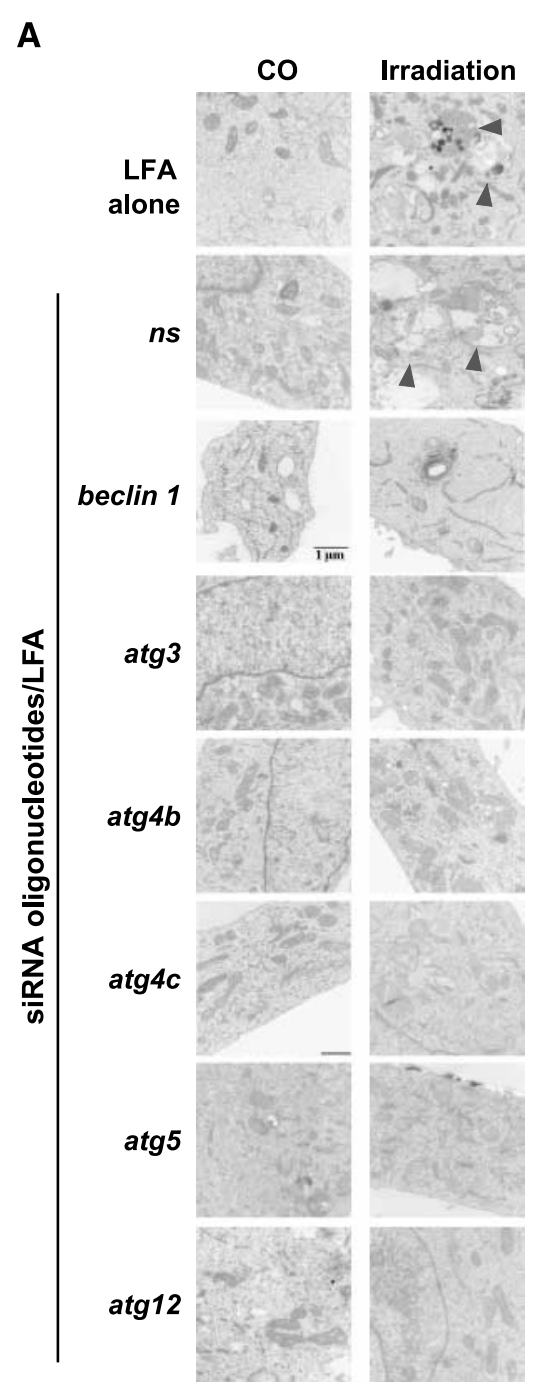

B

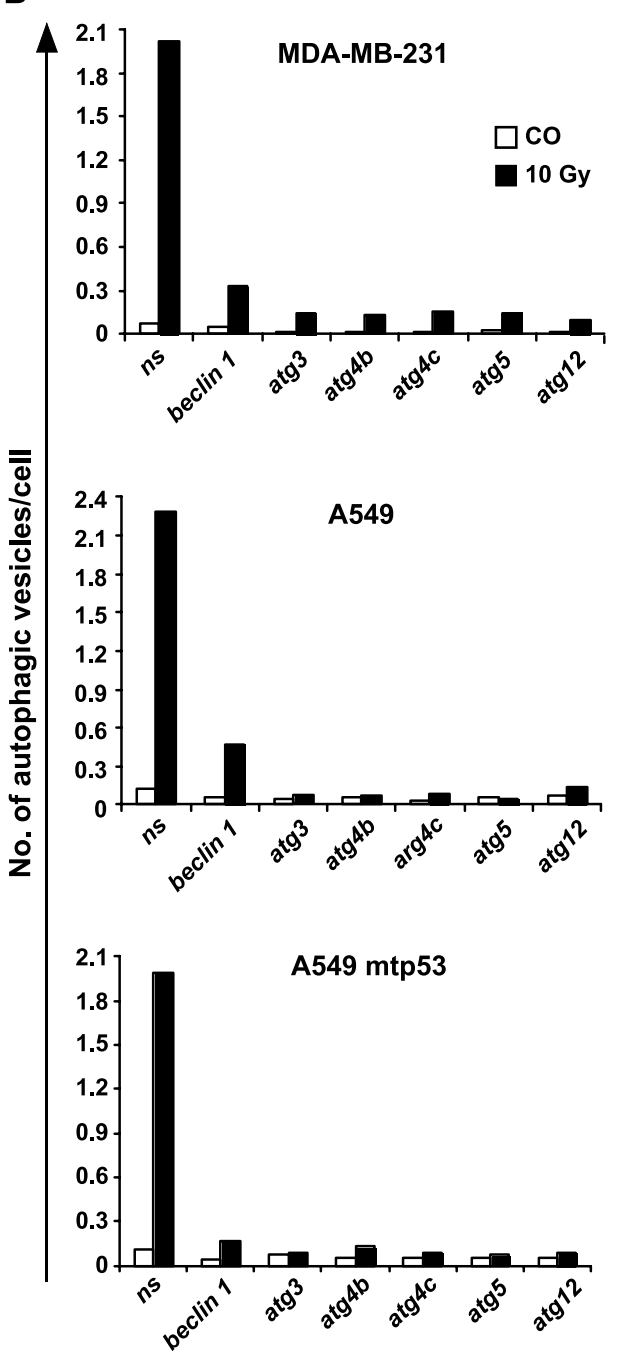

and radiosensitized the radioresistant HTB43 and MDA-MB-231 cells, radioprotection by inhibition of atg3 was observed in sensitive A549 cells with wild-type p53. Similarly, inhibition of atg $4 c$ led to a higher survival rate of HTB43 and A549 cells with wild-type or mutant $p 53$. Although inhibition of atg12 radiosensitized MDA-MB-231 cells as already seen in the experiments described above, it radioprotected HTB43 and A549 cells with wildtype or mutant $p 53$. No opposite effects were seen in clonogenic survival between A549 cells with wild-type or mutant $p 53$. However, A549 cells with mutant $p 53$ are slightly more radioresistant as parental cells, as expected, because defective $p 53$ may prevent apoptosis. Also, the radioprotective effect of specific target-siRNA constructs was more pronounced in cells with mutant $p 53$, suggesting that survival in response to blocked autophagy is stronger in a situation of prevented apoptosis. Overall, our results suggest that inhibition of atg 3 and atg $4 b$ genes may be suited for radiosensitization of resistant but not of sensitive carcinoma cells in our setting.

To examine the response of inhibited autophagy in a model of parental and acquired intrinsic therapy resistance, we established a resistant subline of SW707 human rectum carcinoma cells by continuous culturing of sensitive SW707 cells in increasing concentrations of cisplatin for six months. These cells acquired cross-resistance toward chemotherapy and radiotherapy as detected by measuring viability after treatment with different concentrations of cisplatin and $\gamma$-irradiation (Fig. 5B). For inhibition of autophagy, target-siRNA oligonucleotides toward beclin 1, atg3, atg $4 b$, and atg5 were transfected in resistant and sensitive SW707 cancer cells; and down-regulation of the respective genes was analyzed by LightCycler-RT-PCR 5 days later (Supplementary Fig. S4). Strong inhibition of RNA expression of all genes was found compared with a transfected nonsense-siRNA oligonucleotide. Therefore, the transduced cells were treated with fractionated irradiation at day 2 after transfection, and the outcome of inhibited autophagy on clonogenic survival of parental and derived resistant SW707 was examined by colony forming assay. As already evident in nonirradiated cells, blocked autophagy lowered basal survival in sensitive cells. As evaluated by the Student's $t$ test, this effect was significant for atg $4 b$, atg5, and the mixed siRNAs. In contrast, inhibition of autophagic genes increased clonogenic survival in resistant cells with significant effects upon inhibition of beclin 1, atg3, atg4, and mixed siRNAs (Fig. 5C). After 
A

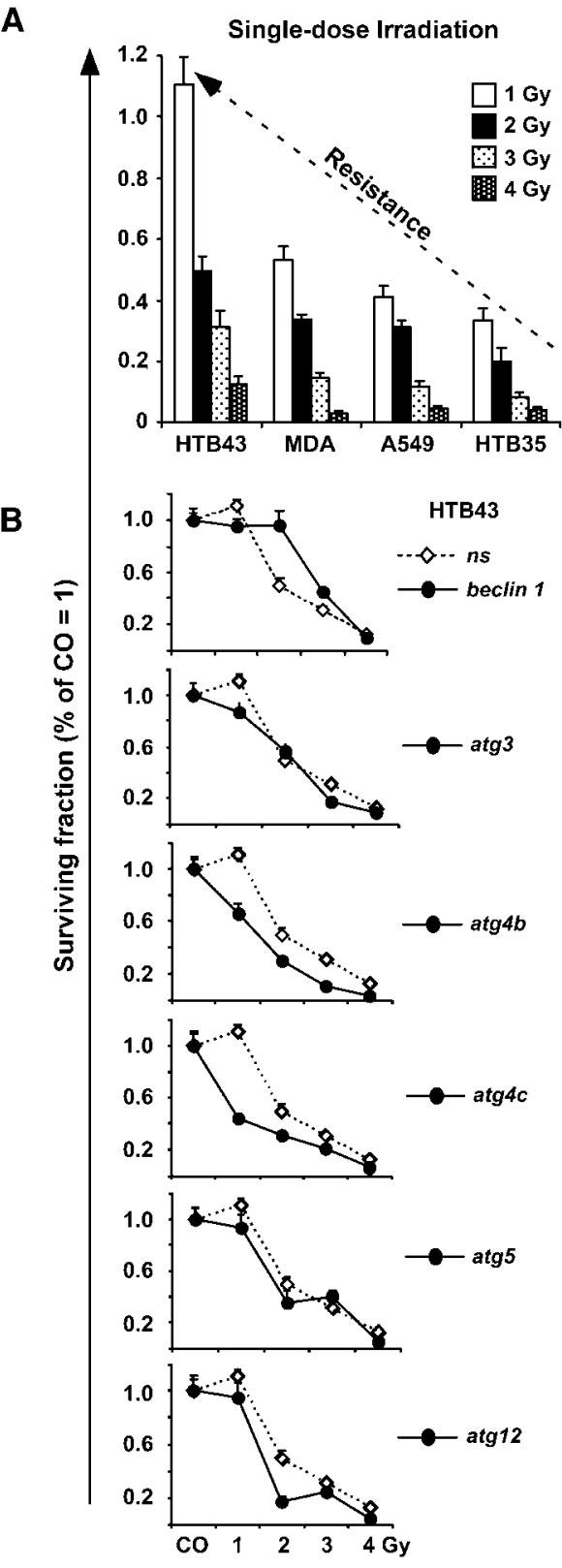

Figure 4. Inhibition of autophagosome accumulation influences clonogenic survival after single-dose irradiation. A, HTB43, MDA-MB-231, A549 with wild-type $p 53$, and HTB35 cells were transfected with nonsense-RNAi oligonucleotides. Two days later, cells were left either untreated or were $\gamma$-irradiated with doses ranging from 1 to $4 \mathrm{~Gy}$ as indicated. Immediately after irradiation, cells were seeded at a density of $200,500,600,700$, or 800 cells per well. Ten days after culturing, cells were fixed in paraformaldehyde and stained with Coomassie Blue. The number of colonies with $>50$ cells was counted under a dissecting microscope, and the percentage of cell survival was calculated (plating efficiency of nonsense siRNA transfected cultures $=100 \%$ relative survival rate). $B$, HTB43 cells were transfected with nonsense-RNAi oligonucleotides ( $n s$ ) or with specific RNAi oligonucleotides as indicated, followed by treatment of the cells as described in A. Columns, pooled mean of three independent experiments; bars, SE.

fractionated irradiation, inhibition of beclin 1, atg5, and a mixture of several autophagy-related genes resulted in significant radiosensitization of parental cells and with an even more pronounced effect of resistant cells. The effect of beclin 1 was less clear because

the respective siRNA significantly radiosensitized parental cells, whereas no strong effect could be observed in the resistant subclone. Therefore, inhibition of autophagy in untreated cells reduces basal survival in sensitive parental cells but leads to enhanced basal clonogenicity of the derived resistant subclone. Upon irradiation, inhibition of atg3, atg $4 b$, atg 5 , or several autophagy genes together strongly reduced clonogenicity of both, sensitive, and resistant rectum carcinoma cells.

\section{Discussion}

In the present study, the therapeutic effect of irradiation-induced autophagy was examined in radiosensitive and radioresistant human carcinoma cells. Because apoptosis plays an only marginal role for radiation-induced cell death or growth arrest in solid tumor cells $(39,40)$, we focused solely on clonogenic survival. Irradiation of two radiosensitive and two resistant cancer cells resulted in all cases in strong and fast induction of vacuolization and double membrane structures. These were detected by conventional electron microscopy, which is the most standard method for monitoring autophagy. Induction of autophagosomes was further shown by transfection of the fluorescent marker protein GFP-LC3 because microtubule-associated protein-1 LC3 is an important component of mammalian autophagosomes $(36,37)$. For the majority of our experiments, cells were irradiated with doses between 1 and $10 \mathrm{~Gy}$, which was sufficient for accumulation of autophagosomes, as also confirmed by Daido and colleagues (41). All cell lines used in this study showed very small autophagosomes, resembling more dots than ring-like structures even with high magnification. Small autophagosomes in our system are within the scope of different possible ranges because the size of autophagosomes depends on the cell type $(42,43)$.

We did not observe an obvious difference between irradiationinduced autophagosomes and the level of resistance of the different used carcinoma cell lines. Also, the level of p53 did not influence the formation of autophagic vesicles upon irradiation because we could not detect a difference between HTB43 pharyngeal cancer, MDA-MB-231 breast cancer, and HTB35 cervical squamous cell carcinoma cells with mutated $p 53$, or A549 lung cancer cells with wild-type $p 53$ and A549 cells in which $p 53$ function was blocked by activation of an Ectysone-inducible mutated $p 53$ construct (mtp53). A recent report showed a direct correlation between damage-regulated autophagy modulator DRAM, a p53 target gene that induces autophagy and promotes $p 53$-mediated death (29). Also, knockdown of atg 5 using specific target-siRNA reveals that autophagy is required for the apoptotic response of $p 53$ and enhanced the ability of alkylating drug therapy to induce tumor cell death $(8,29)$. However, because apoptosis upon irradiation contributes only minor to the therapeutic effect in solid tumor cells $(39,40)$, this may have hindered us from detecting a correlation between the $p 53$ status and accumulation of autophagosomes in our experimental setting.

Prior studies have led to conflicting views of the role of autophagy in cancer therapy. Suppression or deficiency of autophagy genes has been shown to enhance tumorigenesis (24), leading to the conclusion that rapidly growing tumors down-regulate autophagy. Consistent with this finding, the beclin 1 gene is monoallelically deleted in many breast cancers and associated with reduced autophagy in the tumor cells (14). These observations suggest that inhibition of autophagy may contribute to a resistant phenotype, or vice versa, that intact autophagy may suppress 
Table 1. Effects of radiation-induced down-regulated autophagy-related genes on clonogenic survival

HTB43

\begin{tabular}{lccccc}
\hline & beclin 1 & $\operatorname{atg} 3$ & $\operatorname{atg} 4 b$ & $\operatorname{atg} 4 c$ & $\operatorname{atg} 5$ \\
\hline $1 \mathrm{~Gy}$ & $\downarrow$ & $\downarrow$ & $\downarrow$ & $\downarrow$ & \\
$2 \mathrm{~Gy}$ & $\uparrow$ & $\downarrow$ & $\downarrow$ & & \\
$3 \mathrm{~Gy}$ & $\uparrow$ & $\downarrow$ & $\uparrow$ & \\
$4 \mathrm{~Gy}$ & $\downarrow$ & $\downarrow$ & $\uparrow$ & $\uparrow$
\end{tabular}

MDA-MB-231

\begin{tabular}{|c|c|c|c|c|c|c|}
\hline & beclin 1 & $\operatorname{atg} 3$ & $\operatorname{atg} 4 b$ & $\operatorname{atg} 4 c$ & $\operatorname{atg} 5$ & $\operatorname{atg} 12$ \\
\hline $1 \mathrm{~Gy}$ & $\downarrow$ & & & & $\downarrow$ & $\downarrow$ \\
\hline 2 Gy & $\uparrow$ & & & $\uparrow$ & & $\downarrow$ \\
\hline $3 \mathrm{~Gy}$ & $\uparrow$ & & & & & \\
\hline $4 \mathrm{~Gy}$ & & & & & & \\
\hline $4 \times 1 \mathrm{~Gy}$ & $\downarrow$ & $\downarrow$ & $\downarrow$ & & & $\downarrow$ \\
\hline
\end{tabular}

HTB35

\begin{tabular}{lccccc}
\hline & beclin 1 & $\operatorname{atg} 3$ & $\operatorname{atg} 4 b$ & $\operatorname{atg} 4 c$ & $\operatorname{atg} 5$ \\
\hline $1 \mathrm{~Gy}$ & $\uparrow$ & $\downarrow$ & $\uparrow$ & \\
$2 \mathrm{~Gy}$ & & & & \\
$3 \mathrm{~Gy}$ & & & & \\
$4 \mathrm{~Gy}$ & & & & \\
$4 \times 1 \mathrm{~Gy}$ & & &
\end{tabular}

A549-wtp53

\begin{tabular}{lccccc}
\hline & beclin 1 & $\operatorname{atg} 3$ & $\operatorname{atg} 4 b$ & $\operatorname{atg} 4 c$ & $\operatorname{atg} 5$ \\
\hline Gy & $\uparrow$ & & & $\downarrow$ \\
2 Gy & $\uparrow$ & & $\downarrow$ \\
3 Gy & $\uparrow$ & & \\
4 Gy & $\uparrow$ & $\uparrow$ & $\uparrow$ & \\
$4 \times 1$ Gy & $\uparrow$ & $\uparrow$ & $\uparrow$
\end{tabular}

A549-mtp53

\begin{tabular}{ccccccc} 
& beclin 1 & $\operatorname{atg} 3$ & $\operatorname{atg} 4 b$ & $\operatorname{atg} 4 c$ & $\operatorname{atg} 5$ & \\
\hline $4 \times 1 \mathrm{~Gy}$ & & $\uparrow$ & $\uparrow$ & $\uparrow$ & $\uparrow$ & $\uparrow$
\end{tabular}

SW707 SENS

\begin{tabular}{ccccccc} 
& beclin 1 & $\operatorname{atg} 3$ & $\operatorname{atg} 4 b$ & $\operatorname{atg} 5$ & mix & \\
\hline $4 \times 1 \mathrm{~Gy}$ & $\uparrow$ & $\downarrow$ & $\downarrow$ & $\downarrow$ & $\downarrow$
\end{tabular}

SW707 RES

\begin{tabular}{cccccc} 
& beclin 1 & $\operatorname{atg} 3$ & $\operatorname{atg} 4 b$ & $\operatorname{atg} 5$ & mix \\
\hline $4 \times 1$ Gy & $\downarrow$ & $\downarrow$ & $\downarrow$ & $\downarrow$
\end{tabular}

NOTE: Specific target-siRNA constructs toward autophagy-related genes or a mix of beclin 1, atg3, atg4b, and atg5 (mix) were transfected in HTB43, MDA-MB-231, HTB35, A549-wtp53, A549-mtp53, SW707-SENS, or SW707-RES as indicated. Enhanced $(\uparrow)$ or reduced $(\downarrow)$ clonogenicity in response to single-dose $(1,2,3$, or 4 Gy) or fractionated irradiation $(4 \times 1 \mathrm{~Gy})$ relative to control nonsense-siRNA. Dark fields, low or no significant effect. 


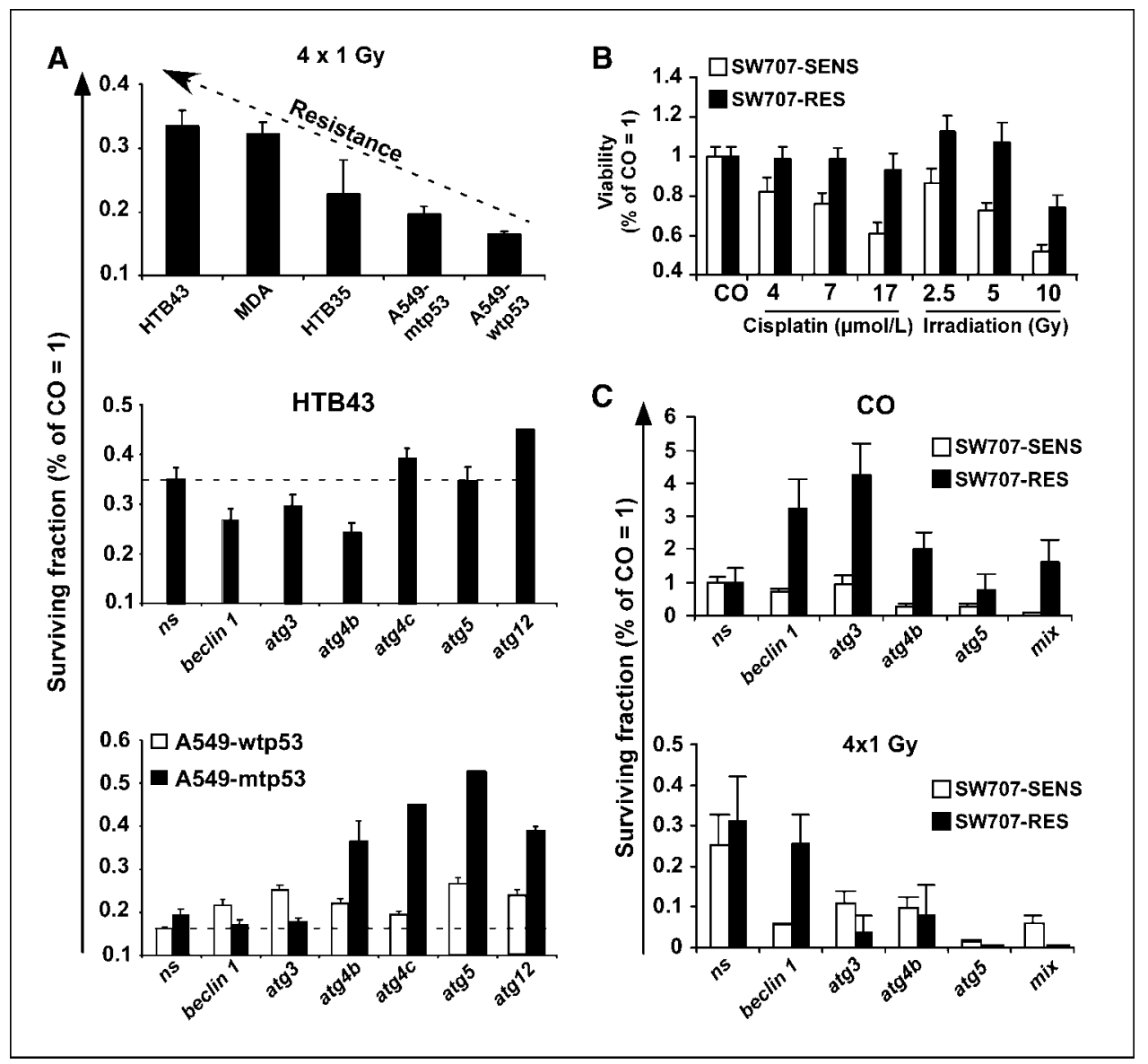

Figure 5. Inhibition of autophagosome accumulation influences clonogenic survival after fractionated irradiation. $A$, HTB43, MDA-MB-231, HTB35, and A549 cells with wild-type (A549-wtp53) or mutant $p 53$ (A549-mtp53) were transfected with nonspecific ( $n s)$ or specific RNAi oligonucleotides as indicated (top). Two days later, cells were left either untreated or were $\gamma$-irradiated with $1 \mathrm{~Gy}$. $\gamma$-Irradiation was repeated every $24 \mathrm{~h}$ four cycles ( $4 \times 1 \mathrm{~Gy})$. After the last treatment, cells were immediately seeded for colony-forming assays and analyzed. HTB43 (middle) and A549 with wild-type or mutant p53 (bottom) were transfected, treated with $4 \times 1 \mathrm{~Gy}$, and analyzed as described above. $B$, parental (SW707-SENS) or a selected resistant subclone (SW707-RES) of human SW707 rectum carcinoma cells were left untreated or were stimulated with cisplatin or $\gamma$-irradiation as indicated. Three days later, viability was determined by MTT assay. SW707-SENS and SW707-RES were transfected with nonspecific or specific siRNA oligonucleotides as indicated. Two days later, cells were either left untreated $(C$, top) or $\gamma$-irradiated with $4 \times 1$ Gy $(C$, bottom). Columns, mean of three independent experiments; bars, SE.

tumor cell outgrowth. In contrast, recent work has shown that autophagy provides an adaptive mechanism to induce resistance to apoptosis for survival of cellular stress as a result of either growth factor or nutrient deprivation $(20,44)$. Because there are several other examples for opposite effects of autophagy in tumor treatment (7), we knocked down beclin 1, atg3, atg4b, atg4c, atg5, and $\operatorname{atg} 12$ by transient transfection of siRNA oligonucleotides in human carcinoma cell lines, which resulted in silencing of the genes up to 8 days. Although our data clearly show that inhibition of each autophagy-related gene can prevent the formation of autophagic vesicles in all cell lines, the effect on clonogenic survival was not consistent and dependent on the cell line and stimulus used. Whereas for example, 1 Gy irradiation of cells with inhibited beclin 1 diminished survival in radioresistant pharyngeal and breast cancer cells, survival in more radiosensitive cervical squamous cell and lung carcinoma cells was enhanced. Using a model of parental sensitive and derived resistant rectum carcinoma cells, inhibition of beclin 1 resulted in further sensitization of parental cells but had no well-defined effect on clonogenicity of the resistant subclone. Another example is inhibition of atg $4 c$, which diminished survival of radioresistant pharyngeal cancer cells after single doses of 1 or $2 \mathrm{~Gy}$, but enhanced survival after fractionated irradiation with $4 \times 1 \mathrm{~Gy}$. Correspondingly, blocked atg5 in radiosensitive lung cancer cells diminished clonogenic survival after single doses of 1 or 2 Gy but enhanced clonogenicity after fractionated irradiation with $4 \times 1$ Gy. Overall, what we can conclude from our experiments is that the majority but not all of inhibited autophagy-related genes resulted in radiosensitization of resistant cells of different origin, whereas the effect on sensitive cancer cells is less clear. By using our model of intrinsic resistance in a rectum carcinoma cell line, we found that although inhibition of autophagy sensitizes resistant cells to radiotherapy, untreated resistant cells even showed a higher basal clonogenicity upon inhibition of autophagy, supporting the hypothesis that inhibition of autophagy may have opposite effects, dependent on the initial cellular state. The observed effect of inhibited autophagy and radiosensitization of resistant cancer cells in our system may be even more pronounced because we cannot ensure that the RNAi effect was total-given that the strength of siRNA-mediated gene silencing is highly dependent on the half-time and abundance of the gene product as well as on the mRNA target sequence and its accessibility (45-47). Thus, the possibility remains that residual protein expression sufficed to induce minimal autophagic activity, although we did not observe formation of autophagosomes upon transfection of RNAi oligonucleotides toward autophagy-related genes. Nevertheless, the absence of autophagosomes does not necessarily indicate a total inhibition of autophagy. Because autophagosomes are only transient in this process, being subsequently turned over in autolysosomes, the observed blocked accumulation of GFP-LC3 could either represent an actual diminished induction of autophagy or an enhanced autophagosome turnover (29). Hosokawa et al. (48) suppose that only a complete suppression of Atg5 leads to fully inhibition of autophagy. As most Atg12-Atg5 conjugate resides in the cytosol, and only a small fraction associates with the isolation membrane, the number of Atg12-Atg5 conjugate functioning in autophagosome formation 
may be very small. Also, Mizushima et al. (49) found a slight autophagy activity in atg5-deficient cells, which he explained by the remaining autophagic ways of microautophagy and chaperonmediated autophagy. To enforce down-regulation of autophagy, we transfected a mixture of four different autophagic siRNAs (beclin1, atg3, atg $4 b$, and atg5) together. By this way, we found complete inhibition of clonogenic survival in radiation-sensitive rectum carcinoma cells compared with parental cells with intact autophagy. In radioresistant rectum carcinoma cells, we did not detect a significant effect on clonogenic survival by a mixture of transfected siRNAs toward autophagy-related genes. After irradiation, inhibition of autophagy genes leads to sensitization of radiosensitive and radioresistant rectum carcinoma cells, although the measured effects were not statistically significant for each inhibited autophagy-related gene. However, the strongest and significant sensitizing effect was found by inhibition of atg 5 siRNA alone or a mixture of beclin1, atg3, atg $4 b$, and atg 5 siRNAs together.

Autophagic vacuoles protect the cells against radiation damage by preventing cytoplasmic acidification, by providing catabolites required for repair processes, and/or by containing toxic molecules. Provided that inhibition of autophagy was total in our system, it may well be that increased needs for catabolite supplies for enhanced DNA repair in radioresistant cells could not be fulfilled in the situation of blocked autophagy, resulting in cell death. In contrast, less catabolites are needed for a slower DNA repair in sensitive cells and might have been still sufficient for radioprotection, despite blocked autophagy. Therefore, moderate formation of autophagic vacuoles in surviving colonies may offer a long-term protection against low-irradiation damage in sensitive but not in resistant cancer cells. In this regard, experiments with Bafilomycin $\mathrm{A}_{1}$ show that inhibition of vacuolar $\mathrm{H}^{+}$-ATPase increased DNA degradation and significantly decreased survival after irradiation of
MCF-7 (human breast adenocarcinoma), LoVo (human colon adenocarcinoma), and LNCaP (human prostate carcinoma) cells (6), which may be classified as radioresistant. In another study of autophagy inhibition, 3-methyladenine led to radiosensitization by repressing repair of radiation-induced DNA double-strand breaks and thus enhancing the antitumor effect of irradiation (48). Corresponding to our results, Carew et al. (44) came to the conclusion that disruption of autophagy by chloroquine treatment is able to sensitize drug-resistant chronic myelogenous leukemia cells to the anticancer activity of a histone deacetylase inhibitor (50). In line with these data, another recent article indicates a function of enhanced autophagy in survival of colorectal cancer cells, suggesting inhibition of autophagy as therapeutic strategy.

In conclusion, inhibition of autophagy may sensitize cancer cells to radiation, whereas basal clonogenicity of untreated resistant cells may be even enhanced by inhibition of autophagy. Our data suggest that inhibition of autophagy in cancer cells may vary dependent on the type of cancer, individual characteristics of cancer cells, microenvironments, and therapeutic treatment. In our system, short time inhibition of autophagy was beneficial to enhance cytotoxicity of radiotherapy in resistant cancer cells.

\section{Acknowledgments}

Received 2/9/2007; revised 12/12/2007; accepted 1/2/2008.

Grant support: The Federal Ministry of Education and Research (I. Herr and H.P. Rodemann), Deutsche Krebshilfe, Tumorzentrum Heidelberg/Mannheim, Stiftung Chirurgie (I. Herr), and the Deutsche Forschungsgemeinschaft (A. Mayer).

The costs of publication of this article were defrayed in part by the payment of page charges. This article must therefore be hereby marked advertisement in accordance with 18 U.S.C. Section 1734 solely to indicate this fact.

We thank Dr. T. Yoshimori for GFP-LC3 cells, U. Mueller and B. Sailer for help in electron microscopy, and Dr. A. Spang for sharing her fluorescence microscope.

\section{References}

1. Codogno P, Meijer AJ. Autophagy and signaling: their role in cell survival and cell death. Cell Death Diff 2005; 12:1509-18.

2. Kroemer G, Jaattela M. Lysosomes and autophagy in cell death control. Nature Rev 2005;5:886-97.

3. Kim R, Emi M, Tanabe K, Uchida Y, Arihiro K. The role of apoptotic or nonapoptotic cell death in determining cellular response to anticancer treatment. Eur J Surg Oncol 2006;32:269-77.

4. Hippert MM, O'Toole P S, Thorburn A. Autophagy in cancer: good, bad, or both? Cancer Res 2006;66:9349-51.

5. Bursch W, Ellinger A, Kienzl H, et al. Active cell death induced by the anti-estrogens tamoxifen and ICI 164384 in human mammary carcinoma cells (MCF-7) in culture: the role of autophagy. Carcinogenesis 1996;17:1595-607. 6. Paglin S, Hollister T, Delohery T, et al. A novel response of cancer cells to radiation involves autophagy and formation of acidic vesicles. Cancer Res 2001;61:439-44.

7. Kondo Y, Kanzawa T, Sawaya R, Kondo S. The role of autophagy in cancer development and response to therapy. Nature Rev Cancer 2005;5:726-34.

8. Amaravadi RK, Yu D, Lum JJ, et al. Autophagy inhibition enhances therapy-induced apoptosis in a Myc-induced model of lymphoma. J Clin Invest 2007:117:326-36.

9. Ogier-Denis E, Codogno P. Autophagy: a barrier or an adaptive response to cancer. Biochim Biophys Acta 2003;1603:113-28.

10. Marino G, Uria JA, Puente XS, Quesada V, Bordallo J, Lopez-Otin C. Human Autophagins, a family of cysteine proteinases potentially implicated in cell degradation by autophagy. J Biol Chem 2003;278:3671-8.

11. Mizushima N, Yoshimori T, Ohsumi Y. Role of the
Apg12 conjugation system in mammalian autophagy. Int J Biochem Cell Biology 2003;35:553-61.

12. Gozuacik D, Kimchi A. Autophagy as a cell death and tumor suppressor mechanism. Oncogene 2004;23. 2891-906.

13. Yorimitsu T, Klionsky DJ. Autophagy: molecular machinery for self-eating. Cell Death Differ 2005;12 Suppl 2:1542-52.

14. Liang XH, Jackson S, Seaman M, et al. Induction of autophagy and inhibition of tumorigenesis by beclin 1 . Nature 1999;402:672-6.

15. Liang XH, Yu J, Brown K, Levine B. Beclin 1 contains a leucine-rich nuclear export signal that is required for its autophagy and tumor suppressor function. Cancer Res 2001;61:3443-9.

16. Kihara A, Kabeya Y, Ohsumi Y, Yoshimori T. Beclinphosphatidylinositol 3-kinase complex functions at the trans-Golgi network. EMBO Rep 2001;2:330-5.

17. Edinger AL, Thompson CB. Defective autophagy leads to cancer. Cancer Cell 2003;4:422-4.

18. Kametaka S, Matsuura A, Wada Y, Ohsumi Y. Structural and functional analysis of APG5, a gene involved in autophagy in yeast. Gene 1996;178:139-43.

19. George MD, Baba M, Scott SV, et al. Apg5p function in the sequestration step in the cytoplasm-to vacuole targeting and macroautophagy pathways. Mol Biol Cell 2000;11:969-82.

20. Lum JJ, Bauer DE, Kong M, et al. Growth factor regulation of autophagy and cell survival in the absence of apoptosis. Cell 2005;120:237-48.

21. Boya P, Gonzalez-Polo RA, Casares N, et al. Inhibition of macroautophagy triggers apoptosis. Mol Cell Biol 2005;25:1025-40.

22. Gonzalez-Polo RA, Boya P, Pauleau AL, et al. The apoptosis/autophagy paradox: autophagic vacuoliza- tion before apoptotic death. J Cell Science 2005;118: 3091-102.

23. $\mathrm{Yu} \mathrm{L}$, Alva A, Su H, et al. Regulation of an ATG7beclin 1 program of autophagic cell death by caspase- 8 Science 2004;304:1500-2.

24. $\mathrm{Qu} \mathrm{X}, \mathrm{Yu} J$, Bhagat $\mathrm{G}$, et al. Promotion of tumorigenesis by heterozygous disruption of the beclin 1 autophagy gene. J Clin Invest 2003;112:1809-20.

25. Yue Z, Jin S, Yang C, Levine AJ, Heintz N. Beclin 1, an autophagy gene essential for early embryonic development, is a haploinsufficient tumor suppressor. Proc Nat Acad Sci U S A 2003;100:15077-82.

26. Pattingre S, Tassa $A, Q u X$, et al. Bcl-2 antiapoptotic proteins inhibit Beclin 1-dependent autophagy. Cell 2005;122:927-39.

27. Hanahan D, Weinberg RA. The hallmarks of cancer. Cell 2000;100:57-70.

28. Beroud C, Soussi T. The UMD-p53 database: new mutations and analysis tools. Human mutation 2003;21: 176-81.

29. Crighton D, Wilkinson S, O'Prey J, et al. DRAM, a p53induced modulator of autophagy, is critical for apoptosis. Cell 2006;126:121-34.

30. Hoyer-Hansen M, Bastholm L, Mathiasen IS, Elling F, Jaattela M. Vitamin D analog EB1089 triggers dramatic lysosomal changes and Beclin 1mediated autophagic cell death. Cell Death Differ 2005; 12:1297-309.

31. Shimizu S, Kanaseki T, Mizushima N, et al. Role of Bcl-2 family proteins in a non-apoptotic programmed cell death dependent on autophagy genes. Nat Cell Bio 2004;6:1221-8.

32. Cordes N, Plasswilm L, Bamberg M, Rodemann HP. Ukrain, an alkaloid thiophosphoric acid derivative of Chelidonium majus L. protects human fibroblasts but 
not human tumour cells in vitro against ionizing radiation. Int J Radiat Biol 2002;78:17-27.

33. Dittmann KH, Gueven N, Mayer C, et al. The presence of wild-type TP53 is necessary for the radioprotective effect of the Bowman-Birk proteinase inhibitor in normal fibroblasts. Radiat Res 1998;150:648-55.

34. Dittmann K, Virsik-Kopp P, Mayer C, Rave-Frank M, Rodemann HP. Bowman-Birk protease inhibitor activates DNA-dependent protein kinase and reduces formation of radiation-induced dicentric chromosomes. Int J Radiat Biol 2003;79:801-8.

35. Zhang C, Kolb A, Mattern J, et al. Dexamethasone desensitizes hepatocellular and colorectal tumours toward cytotoxic therapy. Cancer Lett 2006;242:104-11.

36. Kabeya $\mathrm{Y}$, Mizushima N, Ueno $\mathrm{T}$, et al. LC3, mammalian homologue of yeast Apg8p, is localized in autophagosome membranes after processing. Embo 2000;19:5720-8.

37. Paglin S, Lee NY, Nakar C, et al. Rapamycin-sensitive pathway regulates mitochondrial membrane potential, autophagy, and survival in irradiated MCF-7 cells Cancer Res 2005;65:11061-70.
38. Liang $\mathrm{C}$, Feng $\mathrm{P}, \mathrm{Ku} \mathrm{B}$, et al. Autophagic and tumour suppressor activity of a noval Beclinl-binding protein UVRAG. Nat Cell Biol 2006;8:688-99.

39. Brown JM, Wouters BG. Apoptosis, p53, and tumor cell sensitivity to anticancer agents. Cancer Res 1999;59: 1391-9.

40. Gudkov AV, Komarova EA. The role of p53 in determining sensitivity to radiotherapy. Nature Rev Cancer 2003;3:117-29.

41. Daido S, Yamamoto A, Fujiwara K, Sawaya R, Kondo S, Kondo Y. Inhibition of the DNA-dependent protein kinase catalytic subunit radiosensitizes malignant gliom cells by inducing autophagy. Cancer Res 2005;65:4368-75 42. Mizushima N. Methods for monitoring autophagy. Int J Biochem Cell Biol 2004;36:2491-502.

43. Mizushima N, Yamamoto A, Matsui M, Yoshimori T, Ohsumi Y. In vivo analysis of autophagy in response to nutrient starvation using transgenic mice expressing fluorescent autophagosome marker. Mol Biol Cell 2004 15:1101-11.

44. Sato K, Tsuchihara K, Fujii S, et al. Autophagy is activated in colorectal cancer cells and contributes to the tolerance to nutrient deprivation. Cancer Res 2007; 67:9677-84.

45. Zamore PD. RNA interference: listening to the sound of silence. Nature Struct Biol 2001;8:746-50.

46. Hammond SM, Caudy AA, Hannon GJ. Post-transcriptional gene silencing by double-stranded RNA. Nature Rev Genetics 2001;2:110-9.

47. Crnkovic-Mertens I, Hoppe-Seyler F, Butz K. Induction of apoptosis in tumor cells by siRNA-mediated silencing of the livin/ML-IAP/KIAP gene. Oncogene 2003;22:8330-6.

48. Hosokawa N, Hara Y, Mizushima N. Generation of cell lines with tetracycline-regulated autophagy and a role for autophagy in controlling cell size. FEBS Letters 2006;580:2623-9.

49. Mizushima $\mathrm{N}$, Yamamoto $\mathrm{A}$, Hatano $\mathrm{M}$, et al. Dissection of autophagosome formation using Apg5deficient mouse embryonic stem cells. Journal Cell Biol 2001;152:657-68.

50. Carew JS, Nawrocki ST, Cleveland JL. Modulating autophagy for therapeutic benefit. Autophagy 2007;3: 464-7. 


\section{Cancer Research}

\section{Blocked Autophagy Sensitizes Resistant Carcinoma Cells to Radiation Therapy}

Anja Apel, Ingrid Herr, Heinz Schwarz, et al.

Cancer Res 2008;68:1485-1494.

\section{Updated version Access the most recent version of this article at: http://cancerres.aacrjournals.org/content/68/5/1485}

Supplementary Access the most recent supplemental material at:

Material http://cancerres.aacrjournals.org/content/suppl/2008/02/25/68.5.1485.DC1

Cited articles This article cites 49 articles, 18 of which you can access for free at:

http://cancerres.aacrjournals.org/content/68/5/1485.full.html\#ref-list-1

Citing articles This article has been cited by 27 HighWire-hosted articles. Access the articles at: /content/68/5/1485.full.html\#related-urls

E-mail alerts Sign up to receive free email-alerts related to this article or journal.

Reprints and To order reprints of this article or to subscribe to the journal, contact the AACR Publications Subscriptions Department at pubs@aacr.org.

Permissions To request permission to re-use all or part of this article, contact the AACR Publications Department at permissions@aacr.org. 